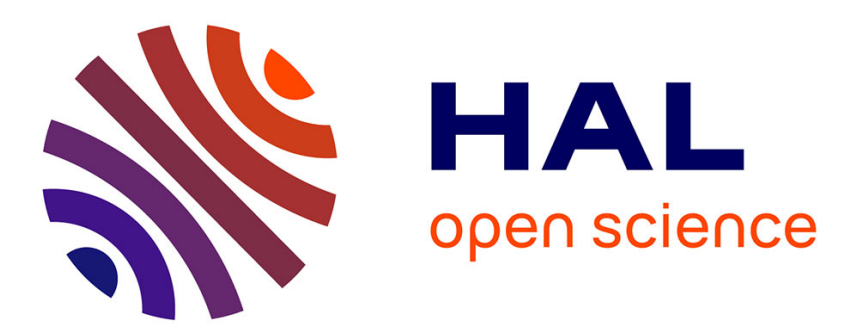

\title{
L'immigration subsaharienne en Espagne vue du sud: entre appel économique et protectionnisme politique
}

Nelly Robin

\section{To cite this version:}

Nelly Robin. L'immigration subsaharienne en Espagne vue du sud : entre appel économique et protectionnisme politique. Migrations Société, 2009, 21 (125), pp.71-90. 10.3917/migra.125.0071 . halshs01057011

\section{HAL Id: halshs-01057011 https://shs.hal.science/halshs-01057011}

Submitted on 21 Aug 2014

HAL is a multi-disciplinary open access archive for the deposit and dissemination of scientific research documents, whether they are published or not. The documents may come from teaching and research institutions in France or abroad, or from public or private research centers.
L'archive ouverte pluridisciplinaire HAL, est destinée au dépôt et à la diffusion de documents scientifiques de niveau recherche, publiés ou non, émanant des établissements d'enseignement et de recherche français ou étrangers, des laboratoires publics ou privés. 


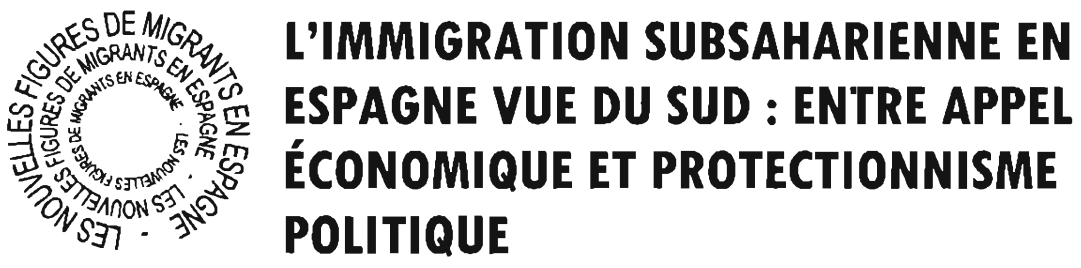

Nelly ROBIN *

Pays traditionnel d'émigration jusqu'aux années 1970, l'Espagne est aujourd'hui l'un des principaux pays d'immigration de l'Union européenne. En $2008,13 \%$ de sa population était née à l'étranger, soit 6 millions de personnes, principalement d'origine européenne $(41 \%)$ et sudaméricaine (33\%); seules $15 \%$ d'entre elles sont nées en Afrique dont $78 \%$ au Maghreb et uniquement $22 \%$ au sud du Sahara ${ }^{1}$.

Bien que la population subsaharienne ${ }^{2}$, essentiellement ouest-africaine ${ }^{3}$, constitue l'une des plus petites communautés immigrées en Espagne (204 393 personnes), elle catalyse depuis quelques années l'attention des politiques, largement relayée par les médias auprès de l'opinion publique.

Étudier l'évolution de l'immigration subsaharienne en Espagne au cours des dix dernières années (1998-2008) peut donc nous aider à comprendre les dynamiques spatiales qui l'animent, du Sud au Nord et entre les pays du Sud, à mieux appréhender les logiques des politiques migratoires qui les stimulent ou les troublent et à discerner les enjeux d'une " coopération pour la régulation et la gestion des flux " migratoires entre l'Afrique et l'Europe. Ces trois questions constituent la trame de notre contribution.

\footnotetext{
Géographe, Centre Population et Développement (CEPED), UMR 196, Institut de recherche pou le développement (IRD), Université Paris Descartes, Institut national d'études démographiques (INED), en accueil au laboratoire MIGRINTER, CNRS, Poitiers.

1. C. Instituto Nacional de Estadistica (INE) ; chiffres du recensemem municipal, padrón, http: /uww.ine.es

2. Ce qualificatif recouvre ici une réalité strictement géographique; nous l'utilisons pour nommer les populations nées au sud du Sahara. Toutefois, cette notion mérite d'être précisée; nous y reviendrons ultérieurement. Selon le champ, politique, journalistique, sociologique, etc., qui la mobilise, elle peut revêtir un sens différent.

3. Ici, l'Afrique de l'Ouest est entendue au sens politique de la Communauté économique des États d'Afrique de l'Ouest (CEDEAO) qui réunit les pays suivants : Sénégal, Gambie, Guinée-Bissau, Guinée, Liberia, Sierra Leone, Cóte d'lvoire, Ghana, Bénin, Togo, Nigenia, Mali, Burkina Faso et CapVert. Pour le moment, la Mauritanie s'en est retirée, mais les autres États lui ont signifié qu'elle peut réintégrer la CEDEAO à tout moment. Nous l'avons donc maintenue dans notre champ ouest-africain.
} 


\section{Une petiłe communaułé venue d'Afrique de l'Ouest}

Les Subsahariens représentent à peine $0,5 \%$ de la population totale et environ $3 \%$ de la population étrangère en Espagne. Numériquement, ils se situent juste après les communautés asiatique (284 283 personnes) et caribéenne ( 263749 ).

Graphique 1 : Répartition de la population ouest-africaine en Espagne selon le pays de naissance, 1998 et 2008

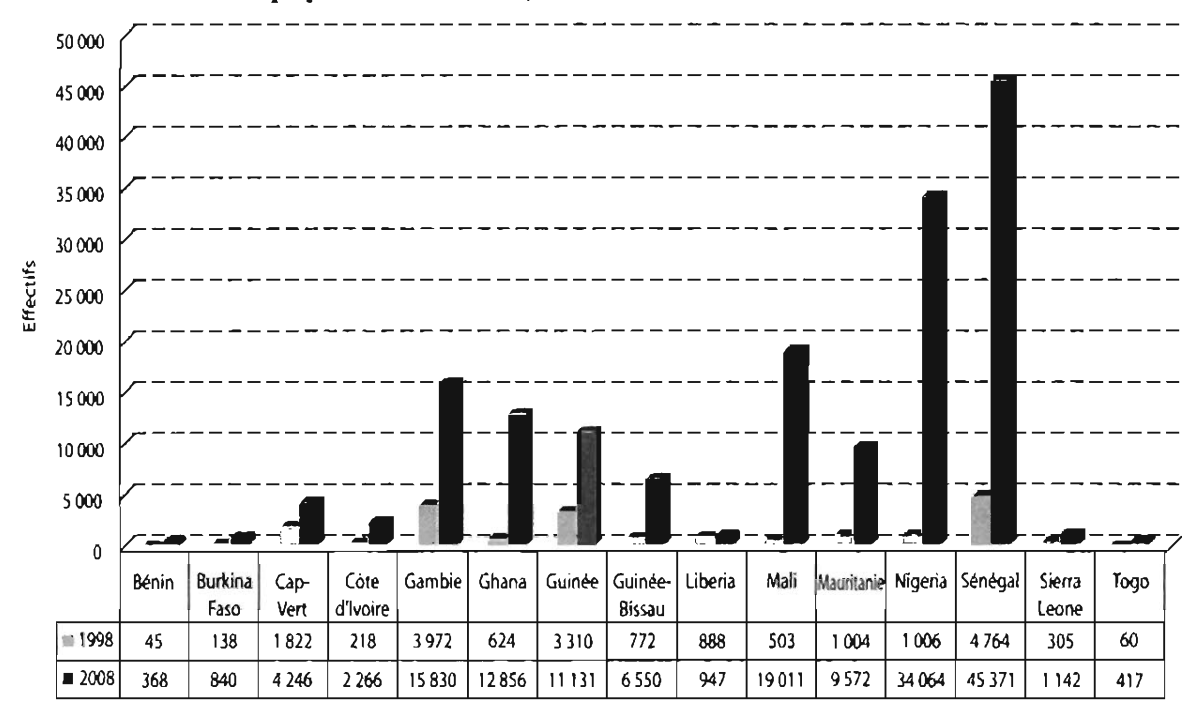

Source : Instituto Nacional de Estadistica, Population par pays de naissance, sexe et année, 1998-2008, http://www.ine.es

$80 \%$ des Subsahariens sont nés dans l'un des pays membres de la Communauté économique des États d'Afrique de l'Ouest (CEDEAO) ${ }^{4}$ ou en Mauritanie. Notre étude sera donc centrée sur la communauté ouestafricaine ${ }^{5}$, estimée à 164611 personnes en 2008 selon les données du

4. Traité de la Communauté économique des États de l'Afrique de l'Ouest, signé à Lagos le 28 mai 1975.

5. Cefte région constitue un espace de libre circulation depuis la signature par les États membres de la CEDEAO du Protocole sur la libre circulation des personnes et le droit de résidence et d'établissement de la CEDEAO, conclu à Dakar le 29 mai 1979

Vol. $21, n^{\circ} 125$

septembre - octobre 2009 
padrón ${ }^{6}$. Plus de $80 \%$ d'entre elles sont nées dans six des 15 pays de l'Afrique de l'Ouest : Sénégal, Nigeria, Mali, Gambie, Ghana et Guinée.

Aujourd'hui, le Sénégal constitue le premier pays d'origine des Africains de l'Ouest présents en Espagne (45 371 personnes), suivi du Nigeria (34 064) - ces deux pays réunissant à eux seuls plus de la moitié de la population née en Afrique de l'Ouest résidant en Espagne - du Mali (19 011 personnes), de la Gambie, du Ghana et de la Guinée, ces trois derniers pays comptant chacun entre 11000 et 15000 personnes.

Cette répartition est bien différente de celle observée à la fin des années 1990, période au cours de laquelle l'immigration ouest-africaine en Espagne émerge réellement ${ }^{7}$. Comptant alors seulement 19431 personnes, soit huit fois moins qu'aujourd'hui, elle provenait essentiellement du Sénégal, de la Gambie et de la Guinée, et plus modestement du Cap-Vert, de la Mauritanie et du Nigeria.

\section{- Des mutations récentes, rythmées par l'évolution des con- trôles aux frontières de l'Europe...}

Ces changements sont représentatifs de l'évolution de l'immigration ouest-africaine dans l'Union européenne au cours des 20 dernières années. Aux relations bipolaires initiales (pays d'origine/pays d'accueil) s'est substituée une diversification des pays d'accueil et une multifonctionnalité des lieux (pôle d'émigration, d'immigration ou de transit). L'immigration en Espagne est le dernier maillon de ce processus en chaîne du nord au sud, puis de l'est à l'ouest de l'Europe.

Au début des années 1990, la France est le premier pays d'acaveil des Africains de l'Ouest en Europe ${ }^{8}$, suivi du Royaume-Uni et de l'Allemagne. Les Sénégalais, les Nigérians et les Maliens privilégient encore des pays d'accueil distincts ; ainsi, les Sénégalais comme les Maliens s'orientent vers la France et les Nigérians vers le Royaume-Uni. Cependant, avec l'entrée en vigueur de la convention d'application de l'accord de Schengen à partir de $1990^{\circ}$, les conditions d'entrée et de séjour des étrangers

6. Recensement municipal publié par l'INE (voir note 1).

7. Toutefois, les premiers flux migratoires datent des années 1980.

8. Cf. ROBIN, Nelly, Adas des migrabions ouest-africaines vers l'Europe, 1985-1993, Paris : Éd. ORSTOM Eurostat, 1996, 109 p. (voir tableau 1, p. 20).

9. En 1985, un accord intergouvernemental relatif à la suppression graduelle des contrôles à leurs frontières communes, dit accord de Schengen, est signé par les trois pays du Benelux, l'Allemagne et la France. Sur cette base, une convention d'application, dite convention de Schengen, est signée en 1990 par les mêmes pays signataires, rejoints ensuite par l'Italie (1990), l'Espagne, le Portugal (1991) et la Grèce (1992). L'espace Schengen comprend aujourd'hui 22 des 27 membres 


\section{Dossier : Les nouvelles figures de migrants en Espagne}

dans les pays traditionnels d'immigration en Europe deviennent de plus en plus restrictives.

Cette évolution conduit les ressortissants des pays ouest-africains, comme ceux des autres pays tiers, vers de nouvelles destinations : I'ltalie puis l'Espagne, dont l'essor économique requiert une main-d'œuvre peu qualifiée, à bas coût et flexible, et qui constituent de nouvelles possibilités de travail pour les migrants. Les pays de l'Europe du Sud, hier source d'émigration, deviennent peu à peu des pays d'immigration.

Ainsi, au milieu des années 1990, l'Italie s'affirme comme le premier pays d'immigration ouest-africaine de l'Union européenne. Les Sénégalais deviennent la première communauté africaine dans la péninsule italienne, suivis par les Égyptiens, les Somaliens, les Ghanéens et les Érythréens ${ }^{10}$. Toute une série de régularisations, organisées entre 1982 et le milieu des années 2000, favorise cette évolution; la communauté sénégalaise, estimée à environ 30000 personnes en 1998, atteint 50000 en $2004^{11}$. Les Ghanéens comme les Nigérians connaissent une évolution comparable, passant respectivement de 15000 à 33000 et de 13000 à 32000 . Mais la loi $n^{\circ} 189$ du 30 juillet 2002 durcit les conditions d'entrée, de séjour et d'asile en ltalie pour les ressortissants des pays tiers. Elle annonce un changement radical dans la politique migratoire italienne dont certaines mesures seront même "l jugées illégitimes par la Cour constitutionnelle en raison des engagements internationaux de l'Italie pour la protection des droits des migrants et des demandeurs d'asile $)^{12}$.

En même temps, "considérant que la voie aérienne [...], lors d'un transit aéroportuaire, constitue un moyen important de pénétration en vue notamment d'un établissement illégal sur le territoire des États membres et qu'il convient de rechercher une amélioration de la maîtrise de cette

de I'Union européenne (UE). L'Irlande et la Grande-Bretagne peuvent participer à tout ou partie de I'“acquis Schengen". En 2009, Chypre pourrait rejoindre intégralement I'acquis Schengen", tandis que la Bulgarie et la Roumanie ne devraient pas y participer totalement avant 2011. La Norvège et I'Islande, pays extérieurs à I'UE, ont un statut d'associé qui leur donne tous les droits sauf celui de participer aux décisions. Depuis décembre 2008, la Suisse est membre de l'espace Schengen. L'adhésion du Liechtenstein est en cours.

10. Il s'agit des communautés supérieures à 10000 personnes. Voir ROBIN, Nelly, Atlas des migrations ouest-africaines vers l'Europe, 1985-1993, op. cit., annexe 1. p. 95.

11. C. ORGANISATION DE COOPÉRATION ET DE DÉVELOPPEMENT ÉCONOMIQUES, OCDE-Stat, Personnes étrangères par nationalité et par pays d'accueil, 1998-2007, http://stats.oecd.org

12. GNISCI, Donata, Mobilités ouest-alricaines et politiques migratoires des pays de l'OCDE, Paris : Éd. CSAO -OCDE, "Cahiers de l'Afrique de l'Ouest", 2008, 145 p. (voir p. 47). 
voie $1{ }^{13}$, le visa de transit aéroportuaire (VTA) est instauré, autorisant ainsi les États membres de l'Union européenne à déroger au principe du "libre passage en transit par la zone internationale des aéroports " établi par l'annexe 9 de la convention de Chicago, seuls les ressortissants des pays tiers étant soumis à ce principe d'exception. D'abord appliqué au Nigeria et au Ghana, le VTA est progressivement étendu à d'autres pays d'Afrique de l'Ouest dont le Burkina Faso, la Côte d'Ivoire, la Gambie, la Guinée, la Guinée-Bissau, le Liberia, le Mali, le Sénégal et la Sierra Leone ${ }^{14}$.

Depuis lors, l'émigration ovest-africaine, se faisant encore essentiellement par voie aérienne, explore d'autres voies et privilégie dans un premier temps la voie terrestre. De nouvelles routes migratoires sont empruntées, l'une des plus actives conjuguant en Afrique de l'Ouest des itinéraires Sud-Nord, partant du golfe de Guinée, et des itinéraires Ouest-Est, depuis les côtes atlantiques, qui convergent vers le Niger, dernière étape avant un transit par la Libye pour rejoindre l'Italie. La Libye devient ainsi un carrefour pour l'émigration subsaharienne vers l'Europe. Des enquêtes effectuées par Ali Bensaâd au cours du premier semestre $2001^{15}$ révèlent que "le niveau minimal des flux de migrants transitant par Agadez [Niger] vers la Libye est de l'ordre de 52000 par an $1{ }^{16}$. Vincent Staub précise que "le Nigeria fournit à lui seul près de la moitié des flux [...] et le Ghana près du tiers, [néanmoins] les flux migratoires prennent racine de plus en plus loin au sud du Sahara, jusqu'au golfe de Guinée et parfois même jusqu'en Afrique centrale ") ${ }^{17}$. Emmanuel Grégoire souligne que le dégel des relations entre la Libye et le Niger, après les tensions des années 1980 et la rébellion tovareg au début des années 1990, a favorisé l'essor de la route du Ténéré ${ }^{18}$.

Face à cette évolution, en 2004 l'Italie promeut la signature d'un "accord de coopération en matière de flux clandestins" avec la Libye ${ }^{19}$, considérée comme une "zone de sas ". Au même moment, l'Union euro-

13. Action commune 96/197/JAl du 4 mars 1996 adoptée par le Conseil sur la base de l'article K.3 du traité de l'Union européenne, relative au régime de transit aéroportuaire, Journal officiel des Communautés européennes $n^{\circ}$ LO63 du 13 mars 1996.

14. C. GROUPE D'INFORMATION ET DE SOUTIEN DES IMMIGRÉS, liste mise à jour en tévrier 2008

15. Enquêtes exhaustives menées directement auprès des transporteurs de migrants et confrontées aux données de la police et à celles de la gendarmerie. Voir STAUB, Vincent, La Libye et les migrations subsahariennes, Paris : Éd. L'Harmattan. 2006, 128 p. (cf. p. 48).

16. Ibidem, p. 48.

17. Ibidem, p. 49.

18. C. GRÉGOIRE, Emmanuel, Touaregs du Niger : le destin d'un mythe, Paris : Éd. Karthala, 1999, et plus précisément le chapitre "Les migrations au Maghreb", pp. 215-243 (voir p. 230).

19. Signé le 12 août 2004. 
péenne se dote d'une agence de surveillance des frontières en Méditerranée, nommée FRONTEX ${ }^{20}$, ayant notamment pour mission " de coordonner la coopération entre les États membres en matière de gestion des frontières extérieures, de les assister pour la formation des garde-frontières nationaux, et de leur fournir l'assistance technique ef l'appui nécessaire pour organiser des opérations de retour conjointes $11^{21}$. L'objectif de FRONIEX est de rechercher en mer les embarcations des candidats à l'immigration en Europe, et surtout, en amont, d'intercepter les bateaux qui embarquent des migrants sur les plages libyennes. À partir d'un centre opérationnel dont le siège est à Tripoli, la mer est surveillée par radar et par satellite jusqu'aux côtes italiennes.

Le déploiement de ces systèmes de surveillance incite les migrants ovest-africains à privilégier la route qui transite par l'Algérie ou la Mauritanie pour rejoindre le Maroc, dans le nord duquel les enclaves de Ceuta et de Melilla deviennent un tremplin vers l'Espagne. L'itinéraire de Jean-Baptiste, reaueilli par Laurent Zamponi en 2004, témoigne de la capacité d'adaptation des migrants à des contextes sans cesse fluctuants : "II a quitté la Casamance [Sénégal] cinq ans auparavant. De la Côte d'lvoire où il a vécu quelque temps, il a essayé de se rendre en Libye via Dirkou [Niger] mais il s'est fait refouler à la frontière. II a ensuite tenté sa chance en passant par Tamanghasset puis Ceuta où ils ont traversé vers l'Espagne à 47 dans une barque à mille euros la place $1{ }^{22}$.

Dans la même logique, les émigrations sénégalaise, nigériane eł ghanéenne glissent de l'Italie vers l'Espagne. Les graphiques 2 et 3 illustrent ce changement, renforcé par l'apparition d'une immigration malienne en Espagne qui, contrairement aux autres, ne succède pas à une période d'immigration en Italie.

20. Agence européenne pour la gestion de la coopération opérationnelle aux frontières extérieures des États membres de l'Union européenne.

21. Site FRONTEX : http://europa.eu/agencies/community_agencies/frontex/index_fr.htm

22. Ce témoignage est extrait d'un rapport de mission de l'ONG Médecins du Monde de novembre 2004 intitulé Rapport de mission exploraloire auprès des populations migrantes en transit par le Niger.

Vol $21, n^{\circ} 125 \quad$ septembre-actobre 2009 
Graphique 2 : L’immigration ghanéenne, nigériane et sénégalaise en Italie de 1998 à 2007

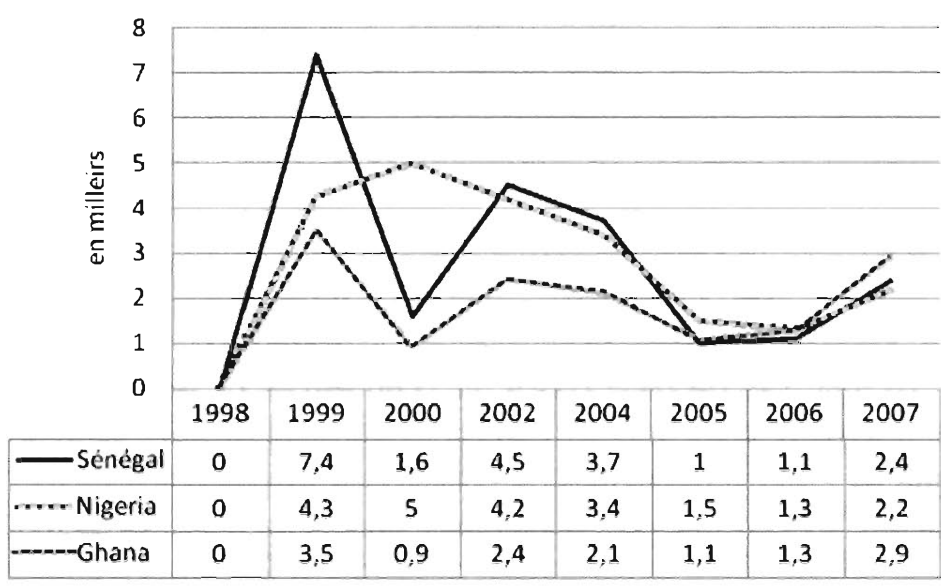

Source : Entrées des étrangers par pays d'immigration, OCDE-Stat.

Graphique 3 : L'immigration ghanéenne, malienne, nigériane et sénégalaise en Espagne de 1998 à 2007

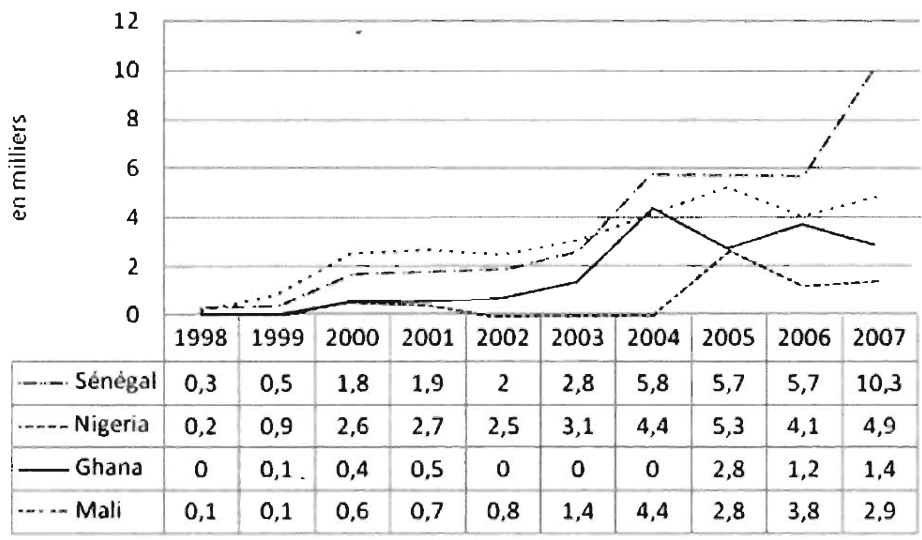

Source : Entrées des étrangers par pays d'immigration, OCDE-Stat. 


\section{- ... favorisées par l'essor de l'économie espagnole et confortées par la crise ivoirienne}

Ce redéploiement dans le sud de l'Europe est favorisé par le dynamisme de l'économie espagnole et soutenu par la procédure de régularisation de $2005^{23}$. Dans ce contexte, la population ovest-africaine immigrée en Espagne augmente régulièrement et ses origines se diversifient, une évolution renforcée par les situations de crise qui se multiplient, parfois s'intensifient et surtout perdurent en Afrique de l'Ouest. II ne s'agit plus seulement d'ajustements conjoncturels en réponse aux sécheresses successives des années 1970 et $1980^{24}$, mais bien d'une réelle mutation des logiques du système migratoire ouest-africain.

La tentative de coup d'État qui a eu lieu le 19 septembre 2002 en Côte d'ivoire, alors premier pays d'immigration africain, constitue un élément clé : plusieurs centaines de milliers de travailleurs immigrés et leurs familles ${ }^{25}$, principalement burkinabés, ghanéens, guinéens ou maliens, ont dû se réfugier dans un pays voisin ou regagner leur pays d'origine, retours qui suscitent de nouveaux candidats à l'émigration. Tous sont à la recherche de possibilités nouvelles, notamment en direction de l'Union européenne où des contacts peuvent être établis plus facilement avec les compatriotes déjà installés.

Ces évolutions participent au redéploiement des ciraulations migratoires régionales dont témoignent notamment les entrées et les sorties des ressortissants ouest-africains enregistrées aux postes frontaliers terrestres de Kidira, entre le Sénégal et le Mali, et de Rosso, entre le Sénégal et la Mauritanie ${ }^{26}$. À partir de 2005, ces deux postes frontaliers s'affirment comme des pôles de transit sur les routes terrestres qui relient l'Afrique subsaharienne à l'Europe soit via le Niger, l'Algérie et la Libye ou le Maroc, soit via la Mauritanie et le Maroc ${ }^{27}$. Les migrants qui empruntent ces routes et transitent par le Sénégal sont gambiens, guinéens, ghanéens,

23. Organisée du 7 février au 7 mai 2005.

24. C. LALOU, Richard, "Les migrations internationales en Atrique de l'Ouest tace à la crise", in : COUSSY Jean : VALIN, Jacques (sous la direction de), Crise et population en Afrique de l'Ouest. Crises éco nomiques, politiques d'ajustement et dynamiques démographiques, Paris : Éd. CEPED, 1996, pp. 345-373.

25. De 500000 à 600000 personnes selon les Nations unies.

26. Données du ministère sénégalais de l'Intérieur.

27. C. GONIN, Patrick; ROBIN, Nelly, "Les routes migratoires par le Sénégal", in : BENSAÂD. Ali (sous la direction de), Le Sahara et le Maghreb, un espace migratoire en devenir, Paris : Éd. Karthala, 2009, pp. 139-170.

Vol. $21, n^{\circ} 125$

septembre - octobre 2009 
nigérians, mais aussi burkinabés, ivoiriens, nigériens et maliens, les migrants sénégalais utilisant aussi ces itinéraires.

Parallèlement, le nombre de ressortissants ovest-africains augmente régulièrement en Espagne. Le graphique 4 révèle l'affirmation de trois espaces d'origine :

* le premier, situé le long du littoral atlantique, est composé du Sénégal et des pays limitrophes tels que la Mauritanie, la Gambie, la GuinéeBissau et la Guinée ;

* le second, à proximité du golfe de Guinée, réunit les deux pays anglophones de la CEDEAO : le Nigeria ef le Ghana;

* le troisième enfin, au centre de l'Afrique de l'Ouest, est constifué du seul Mali, pays frontalier à la fois du Sénégal et de la Côte d'lvoire. La croissance du nombre de Maliens, plus nombreux à partir de 2004, est liée à l'évolution de l'émigration depuis leur pays d'origine mais aussi à l'arrivée en Espagne de Maliens vivant auparavant sans papiers et sans travail en France ou en Belgique, attirés par le dynamisme de l'économie espagnole et espérant bénéficier de la procédure de régularisation organisée en 2005.

Graphique 4 : Évolution de la population ouest-africaine en Espagne selon le pays de naissance, 1998-2008

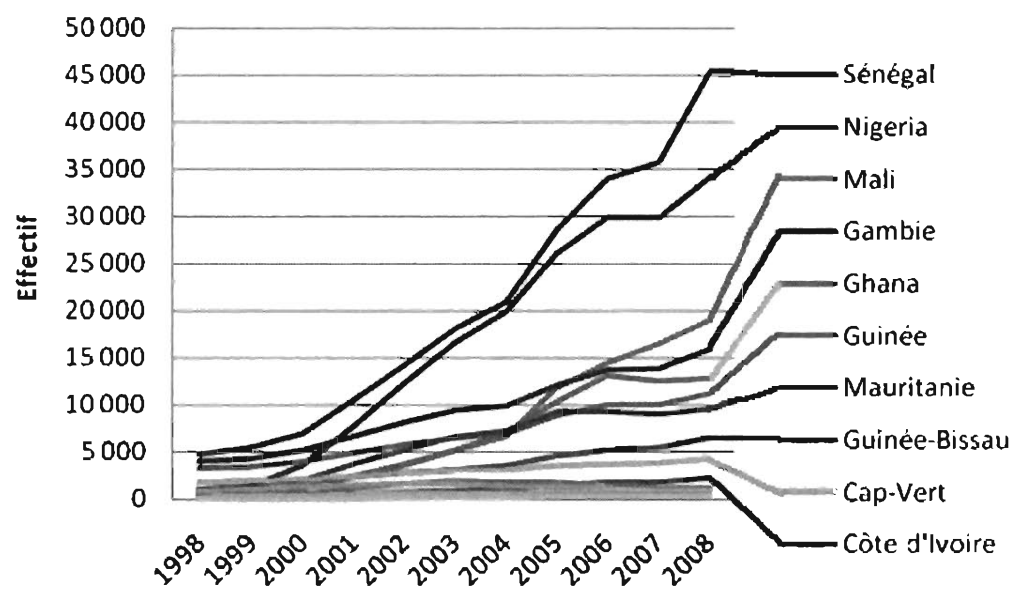

Source : Entrées des étrangers par pays d'immigration, OCDE-Stat. 
Cefte évolution est associée à une réelle diversification de l'émigration ouest-africaine vers l'Espagne. En 1998, six groupes nationaux ouestafricains totalisaient plus de 1000 personnes, 11 dépassent cet effectif en 2008 ; parmi ceux-ci, six comptent plus de 11000 ressortissants. Ces mutations traduisent l'attractivité de l'Espagne en plein essor économique.

\section{Les routes de l'Atlantique, une nouvelle alternative spa- tiale et économique}

Dans ce contexte, le drame de Ceuta et de Melilla ${ }^{28}$, enclaves espagnoles situées dans le nord du Maroc, incite les migrants ovest-africains à explorer les voies maritimes situées plus au sud du Sahara. Ainsi, en huit mois, d'octobre 2005 à mai 2006, les lieux d'embarquement se sont déplacés de la Méditerranée à l'Atlantique, glissant progressivement de Dakhla, dans le sud du Maroc, à Nouadhibou, en Mauritanie, jusqu'à Ziguinchor, dans le sud du Sénégal, la route vers les îles Canaries étant dès lors privilégiée.

Les propos de Moussa ${ }^{29}$, qui a tenté d'émigrer vers l'Espagne en 2006, témoignent de ce redéploiement spatial le long des côtes africaines: "L'émigration maritime a commencé je pense fin 2005 en Mauritanie, après Saint-Lovis ${ }^{30}$, après Dakar. Quand cela s'est ébruité, ils ont commencé à Ziguinchor $11^{31}$.

Le récit de Boubacar, peintre en bâtiment, parti de Casamance en 2006, illustre les étapes du processus : "I'ai essayé par deux fois de passer par le Maroc sans succès. C'est en Mauritanie, alors que je faisais la pêche, que des jeunes comme moi sont partis en Espagne et leurs échos nous parvenaient à chaque fois comme quoi ils sont rentrés dans ce pays [...]. J'ai essayé de passer par deux fois, et chaque fois c'est la marine marocaine qui nous faisait revenir. Je suis alors revenu au Sénégal [...] ; de

28. À l'automne 2005, au nord du Maroc, lors de tentatives collectives pour pénétrer dans les enclaves espagnoles de Ceuta et Melilla, une dizaine de personnes originaires d'Afrique noire sont abattues.

29. Pour des raisons de confidentialité, tous les prénoms de nos interlocuteurs ont été modifiés.

30. Ville située dans le nord du Sénégal.

31. Les paroles des migrants transcrites dans cette contribution ont été recueillies au Sénégal auprès de candidats à l'émigration qui ont tenté de rejoindre les îles Canaries en pirogue depuis les côtes ouest-africaines: certains ont connu des accidents au cours de leur voyage et sont revenus dans leur quartier ou leur village d'origine, d'autres ont été "réadmis" après un séjour dans les camps de rétention des îles Canaries. Les entretiens (40) se sont déroulés de juillet à août 2007 dans la région de Dakar et en Casamance. Ce travail a été réalisé dans le cadre d'un programme lancé par l'UNESCO.

Vol. $21, n^{\circ} 125$

septembre - octobre 2009 
là des parents et amis partis par $D$. [un village de Casamance] $m$ 'ont souvent téléphoné pour me demander de tenter cette voie. J'ai fini par me décider. La pirogue contenait 150 personnes [...] ; plusieurs nationalités composaient le groupe : des Sénégalais, des Bissau-Guinéens, des Gambiens, des Guinéens de Conakry et des Maliens "I.

Cette émigration par voie maritime révèle une demande sociale qui s'accroît et se diversifie au Sénégal en particulier, et de manière plus générale dans la plupart des pays ouest-africains. Les pêcheurs, profondément affectés par une crise halieutique sans précédent, vont mettre leur "savoir-naviguer" au service d'une population en attente d'occasions, parfois sans tradition d'émigration: "Depuis longtemps, i'ai voulu partir en Europe, mais la difficulté d'obtenir un visa ou le passage très pénible du Maroc ont tempéré mon ardeur. Lorsqu'est venue la solution de passer par la mer, i'ai commencé à me dire que l'heure était venue pour moi avec mes maigres moyens de partir, bien que très risqué et très dangereux ", explique Fodé, âgé de 35 ans et père de deux enfants.

La plupart des candidats au départ sont des hommes adultes et actifs, âgés de 25 ans et plus, même si parfois quelques mineurs les accompagnent ${ }^{32}$, qui exercent une activité essentiellement dans les secteurs de la pêche, de l'artisanat, du commerce ou de l'agriculture ${ }^{33}$.

Ainsi, les pirogues, pour reprendre le terme des migrants, constituent une nouvelle alternative pour ces chefs de famille, époux ou fils, confrontés à des difficultés économiques grandissantes et inquiets face à une situation politique pleine d'incertitudes.

Le récit d'lbrahima exprime ce désarroi : "Je suis soutien de famille. J'ai été jusqu'en terminale au lycée, mais après les échecs répétés [au baccalauréat], je suis parti faire du commerce; je me suis débrouillé pour fonder une famille, mais c'est dur de s'en sortir. Alors, l'idée de partir à l'étranger a germé petit à petit, jusqu'au jour où je suis parti par les pirogues 1$)$.

32. Sur un total de 931 mineurs accueillis par les dispositifs de protection insulaires, presque la moitié (453) ont déclaré être sénégalais. Les mineurs marocains, principalement originaires du sud du pays, constituent la deuxième nationalité (290), suivis des Maliens (115). Les autres sont d'origine ouest-africaine (73); ils sont principalement mauritaniens, gambiens ou guinéens. Voir ROBIN, Nelly; SENOVILLA, Daniel, "Unaccompanied and separated children's migration from Senegal to Spain", in : SENOVILA, Daniel (sous la direction de), Migrating alone. Unaccompanied and separated children's migration in Europe, Paris : UNESCO. International Migration Section, Social and Human Sciences, à paraitre.

33. D'après les entretiens auprès de migrants de retour ou "réadmis" et des données judiciaires du Sénégal (Registre des plaintes et des procès-verbaux du Sénégal). De 2006 à 2007, environ 2000 personnes ont été arrêtées au moment de leur départ. 
Fodé ajoute : " Dans notre zone, l'immigration à l'origine n'étaił que l'affaire d'une communauté qui sont les Manjaques ${ }^{34}$. Ces derniers avaient comme chemin l'avion avec visa. Mais ces derniers temps, la crise en Casamance, qui a entraîné la pauvreté dans les familles, une agriculture sans moyen, une pêche mourante obligea les autres ethnies à faire comme les Manjaques qui nous émerveillaient avec les belles constructions ef un meilleur pouvoir d'achat 11.

Pour partir, ces hommes n'hésitent pas à vendre leur outil de travail ou une partie du patrimoine familial : "L'argent qui m'a permis de faire le voyage est le fruit de la vente de trois de nos boufs du cheptel familial II, précise Paul.

Le récit d'Alpha, qui habite Ziguinchor, souligne que la situation est comparable pour ceux qui vivent en milieu urbain : II'étais photographe [...] ; en accord avec la famille de tenter ma chance [...], i'ai vendu mon matériel de studio de photo, une machine de recharge de batteries, une télévision plus DVD, une caméra de reportage et ma mobylette "1.

Parfois, les mères vendent elles-mêmes leurs bijoux ou offrent à leur fils le gain de leur tontine $e^{35}$. Les candidats à l'émigration peuvent aussi être mandatés par leur communauté villageoise. Dans son roman intitulé Mbêkê mi : à l'assaut des vagues de l'Atlantique ${ }^{36}$, l'écrivain sénégalais Abasse Ndione relate l'histoire d'une communauté rurale qui, avec le concours de ses ressortissants émigrés en ltalie, se mobilise pour permettre aux "jeunes désœuvrés dans les villages " de rejoindre les îles Canaries en pirogue, une fiction très proche de la réalité : "L'imam de la grande mosquée, par téléphone, avait expliqué tout cela au président des Associations des ressortissants de la communauté rurale en Europe qui résidait en Italie. Le président avait demandé un délai de réflexion d'une semaine, le temps de consulter ses collègues des autres associations vivant dans les pays européens. La réponse leur était parvenue avant-hier. Elle était positive. Les 16 millions nécessaires au prix du voyage de 40 jeunes, 10 choisis comme convenu dans chaque village, avaient été envoyés à l'imam au bureau de la Western Union à Bakel II.

34. Ethnie du sud de la Casamance.

35. Le principe de la tontine sénégalaise est simple : chaque semaine une mère de famille donne une somme fixe (généralement entre 500 et 1000 francs CFA) et mensuellement une ou plusieurs familles se voient attribuer à tour de rôle une somme importante.

36. NDIONE, Abasse, Mbêkê mi : à l'assaut des vagues de l'Atlantique, Paris : Éd. Gallimard, 2008, 82 p. (voir p. 14).

Vol. $21, n^{\circ} 125$ septembre - octobre 2009 
Ailleurs, la solidarité s'est exprimée entre jeunes d'un même quartier ; ainsi, à Yakha, un village de pêcheurs, à proximité du centre de Dakar, les jeunes ont construit une pirogue pour permettre "laux fils des familles sans ressources de partir aussi 1 .

Les pirogues peuvent réunir des migrants originaires de toutes les régions du Sénégal, comme le confirme l'expérience de Yoro, parti d'une plage dakaroise: "Nous étions 105 passagers. Ils venaient de Yoff [Dakar], Tambacounda, Kedougou [Sénégal oriental], Kolda, Ziguinchor [Casamance], Thiès, Touba, Kébèmer [bassin arachidier]... ".

Ces nouveaux migrants ne recourent pas à des réseaux organisés, l'information se diffusant simplement par les relations familiales ef professionnelles ou par le bouche à oreille entre amis : " Je suis un commerçant, explique Youssouph, je fais des va-et-vient entre $D$. [un village de Casamance] et Ziguinchor pour aller acheter des marchandises. Pendant longtemps je le faisais avec des jeunes comme moi, mais au fur et à mesure que je partais à Ziguinchor, je me rendais comple que mes camarades s'en allaient en Espagne, et chaque fois que je demande, on me disait qu'ils ont pris la mer. Je décidais alors de faire comme eux 11.

Issa, capitaine de pêche, devenu selon sa propre expression "I capitaine de pirogue ", évoque "les difficultés liées à l'obtention du visa, à la longueur du trajet du Maroc qui ont amené les gens à choisir la voie des pirogues [...] moins chère et qui ne pose pas de problème de papiers 11.

Selon le ministère de l'Intérieur espagnol, 609 embarcations et 30246 migrants ont atteint les îles Canaries en 2006. La plupart d'entre eux seraient partis du Sénégal.

Pour Issa, "l l'émigration est aujourd'hui considérée comme la voix du salut pour sortir les paurres de leur situation 11. Au-delà de cette opinion personnelle, tous les éléments exposés précédemment expriment une grande détresse économique et un désarroi social profond; les candidats à l'émigration maritime et leur entourage, familial, professionnel ou villageois, doutent de l'avenir, pour eux-mêmes et leurs enfants. Sans perspective de changement, "en bons chefs de famille " selon leur propre expression, ils préfèrent tenter l'aventure, conscients de tous les dangers que présente la traversée en "pirogue". 


\section{Une "coopération" espagnole, emblématique de la poli- tique migrałoire européenne}

À l'évidence, les candidats à l'émigration actuels n'ont pas le même profil que ceux des années 1960 ; ils ont un niveau d'instruction plus élevé et souvent un métier entre les mains. Leur départ traduit le désarroi de toute une partie de la population, notamment urbaine, jeune et relativement qualifiée, dont les attentes n'ont pas été prises en compte par les politiques de développement. L'accord de Cotonou entre les États membres de l'Union européenne et le Groupe des États d'Afrique, des Caraibes et du Pacifique (ACP), signé le 23 juin 2000 et ratifié le ler avril 2003, reconnaît implicitement cette situation, en précisant que désormais la question des migrations doit être pleinement intégrée aux "I stratégies visant à réduire la pauvreté, à améliorer des conditions de vie et de travail, à créer des emplois et à développer la formation " afin de contribuer "à long terme à normaliser les flux migratoires I). Dans ce contexte, la détermination des nouveaux émigrants par voie maritime interpelle avant tout l'efficience des politiques de développement de ces dernières décennies.

Cependant, pour y répondre, très vite les États européens se sont placés sur un terrain symbolique : les arrivées des pirogues sur les côtes des Canaries, très médiatisées et associées à des discours politiques alarmistes, ont donné à l'opinion publique européenne le sentiment d'une " vague d'immigration subsaharienne sans précédent $" 1$ déferlant sur les côtes espagnoles. Un regard plus objectif aurait permis d'apprécier cet événement à sa juste mesure.

En 2006, 30000 Subsahariens ont atteint les côtes des îles Canaries; parmi eux, de 7000 à 10000 ont été "réadmis" dans leur pays d'origine. Or, la même année, l'Espagne, en quête de main-d'œuvre, a vu sa population étrangère augmenter de $10 \%$, soit de 446136 personnes, essentiellement originaires d'Europe et d'Amérique du Sud. Ainsi, l'idéologie politique a prévalu sur l'objectivité pour une appréciation raisonnée de l'immigration par voie maritime, certes spectaculaire dans la forme et assortie de drames humains mais numériquement limitée.

Dans ce contexte, les accords passés de 2006 à 2008 entre l'Espagne et la Gambie, le Ghana, la Guinée, la Guinée-Bissau, le Mali, la Mauritanie, le Nigeria et le Sénégal pour une "gestion concertée des flux " migratoires sont emblématiques de la politique sécuritaire promue par les pays européens. L'essentiel des efforts déployés porte sur une militarisation des dispositifs de contrôle aux frontières des pays ouest-africains, l'objectif étant "d'intercepter les pirogues d'émigrés clandestins " suspec-

Vol 21, $n^{\circ} 125 \quad$ septembre-octobre 2009 
tées de faire route vers l'archipel espagnol des Canaries. L'identification des candidats à l'émigration vers le territoire européen et la responsabilité du résultat de ces opérations sont déléguées aux autorités sénégalaises; dès lors, l'aide au développement est implicitement assujettie à la "qualité" des résultats obtenus.

\section{- Militarisation des contrôles et pénalisation des migrants}

Ainsi, en juin 2006, à la demande de l'Espagne, FRONTEX $X^{37}$ étend son "dispositif de contrôle de l'émigration clandestine " aux eaux de l'Atlantique Nord, d'abord au large de la Mauritanie, puis du Sénégal ${ }^{38}$. Ces nouveaux accords signés avec les autorités mauritaniennes et sénégalaises prolongent celui signé entre l'Espagne et le Maroc en février 2005 ; tous prévoient de " renforcer les liens entre les forces de sécurité des deux pays, en matière de lutte contre l'immigration clandestine, les réseaux de trafic des êtres humains, le contrôle maritime des activités de pêche et même dans le domaine de la détection de trafic de drogue $11^{39}$. En 2008, l'Espagne a signé trois nouveaux accords avec la Gambie, la Guinée-Bissau et la Guinée afin d'étendre le périmètre de surveillance de FRONTEX.

Ces dispositifs qui visent à renforcer l'intégrité territoriale de l'Europe ont conduit à une pénalisation erronée des candidats à l'émigration. Entre 2006 et 2007, plus de 1500 Sénégalais ${ }^{40}$ ont été interpellés sur les côtes ou dans les eaux territoriales de leur pays, puis poursuivis, jugés et condamnés pour " émigration clandestine " ou " immigration clandestine ", c'està-dire pour une action que, en toute rigueur, ils n'auraient pu commettre que s'ils avaient franchi une frontière ; ils sont donc poursuivis pour une

37. FRONTEX est une agence européenne qui a pour mission de coordonner a la sécurité aux trontières en assurant la coordination des actions des Étals membres dans le cadre de la mise en ceuvre des mesures communautaires relatives à la gestion des frontières extérieures n. En outre, le financement de cette agence dépend d'une subvention communautaire, inscrite à cel effet dans le budget général de l'Union européenne. alimenté par les États membres. Ainsi, tout accord bilatéral signé par l'un des États membres pour le déploiement du dispositif FRONTEX aux frontières d'un pays tiers engage l'Union européenne.

38. En juin 2007, les autorités espagnoles et sénégalaises ont signé un nouvel accord qui prolonge d'un an les opérations de FRONTEX au large des côtes du Sénégal : il a été de nouveau prorogé par un accord signé par le ministre de l'Intérieur sénégalais et son homologue espagnol en mai 2008.

39. ROBIN. Nelly, "La CEDEAO, un espace de libre circulation, poste-frontière avancé de l'espace Schengen", in : TRÉMOLIĖRES, Marie (sous la direction de), Les enjeux régionaux des migrations ouest-africaines. Perspectives africaines et européennes, Paris : OCDE - CSAO, "Cahiers de l'Afrique de l'Ouest", 2009, pp. 148-164 (voir p. 156)

40. D'après une source judiciaire. 
intention qui leur est prêtée, en l'occurrence le projet d'émigrer en Espagne ${ }^{41}$.

Cette situation paradoxale révèle les enjeux, pour les pays du Sud, d'une traduction dans leur droit positif de textes intemationaux de droit pénal visant la ciraulation des personnes. Incité par les pays européens et certaines instances internationales, le Sénégal a, par la loi 2005-06 ${ }^{42}$, introduit dans son droit interne les dispositions du Protocole contre le trafic illicite de migrants par terre, air et mer (2003), associé à la convention des Nations unies contre la criminalité transnationale organisée (2000). Ce recours à des textes de droit pénal pose une question fondamentale : celle de la continuité ou de la rupture du principe de la liberté d'émigrer consacré par l'article 13 de la Déclaration universelle des droits de l'homme ${ }^{43}$ et repris par l'ensemble des textes internationaux ou régionaux de droits humains en matière de migration ou d'asile qui lui sont postérieurs.

\section{- Des retours forcés sans ressource}

Parallèlement, en 2006 et 2007 , la Gambie, la Guinée et la GuinéeBissau, notamment, ont signé avec l'Espagne des accords de réadmission. Pour le moment, leur application reste difficile. Un diplomate en poste à Dakar reconnaît que l'accord avec la Gambie a été signé " pour la forme mais sans effet d'exécution $1{ }^{44}$. De son côté, le Sénégal a accepté plus de 7000 réadmissions alors que le texte qui les prévoit fait encore l'objet de négociations. Selon un autre diplomate européen rencontré au Sénégal, les rapatriements ne font pas l'objet d'une programmation régulière. lls sont organisés "au coup par coup " : "Les Sénégalais sont en première ligne non pas en raison de la signature d'un accord de réadmission, mais en raison de la disponibilité des autorités sénégalaises pour des ra-

41. Cf. NDIAYE, Mandiogou: ROBIN, Nelly, "Le migrant criminalisé, le temps d'une traversée L'exemple de l'émigration récente par voie maritime depuis les cótes sénégalaises", in : TRÉMOLIĖRES. Marie (sous la direction de), Les enjeux régionaux des migrations ouestafricaines. Perspectives africaines et européennes, Paris : OCDE - CSAO, "Cahiers de l'Afrique de l'Ouest", 2009, pp. 185-206.

42. Loi du 29 avril 2005, entrée officiellement en vigueur le 10 mai 2005. Le texte comporte quatre chapitres. Le premier est consacré à la traite des personnes et l'exploitation de la mendicité d'autrui et le second au trafic de migrants; le troisième définit la procédure et le quatrième aborde la question de la protection des victimes et témoins.

43. Article 13: " 1. Toute personne a le droit de circuler librement et de choisir sa résidence à l'intérieur d'un État. 2. Toute personne a le droit de quitter tout pays, y compris le sien, et de revenir dans son pays".

44. Les témoignages recueillis auprès des diplomates européens en poste au Sénégal l'ont été sous couvert de l'anonymat.

Vol. $21, n^{\circ} 125 \quad$ septembre-octobre 2009 
patriements ponctuels et discrets; ainsi, en $48 \mathrm{~h}$, six vols de trois avions peuvent être organisés entre minuit ef $8 \mathrm{~h}$ du matin 11 .

Toutefois, ces rapatriements devaient être assortis de projets créateurs d'emploi et générateurs de revenus pour inciter les retours volontaires, mais la réalité est tout autre : "Une nuit ils sont venus nous prendre pour nous amener à l'aéroport "1, explique Falilou, placé quelques jours dans un camp des îles Canaries avant d'être rapatrié au Sénégal. " Nous pensions qu'on allait à Barcelone. On a su qu'on nous rapatriait quand ils nous ont donné $50 €$ parce que ceux qui nous avaient devancés avaient téléphoné de Dakar pour nous informer qu'ils n'ont pas été à Barcelone mais au Sénégal et qu'on leur avait remis $50 €$ dans l'avion [...]. Nous avons atterri à 7 heures du matin à l'aéroport Léopold Sédar Senghor. À la descente, il y a eu les formalités ovec les empreintes, adresses, numéros de téléphone; ils nous ont donné 10000 francs CFA [15 €] et chacun est rentré de son côté ). Elicio précise " qu'ils avaient dit qu'ils allaient nous appeler, mais rien jusqu'à présent 11, soit un an plus tard. À plusieurs reprises, différentes associations de "réadmis" ont relayé ce désarroi, étonnées d'être sans nouvelles des 14 millions d'euros de la coopération espagnole destinés à des "projets de développement" dans les régions d'émigration, selon l'annonce faite par les autorités sénégalaises.

En réalité, beaucoup de "réadmis" n'ont pas osé rentrer chez eux : "I J'avais honte, dit Abdou. Ma famille a vendu nos bouts pour payer les 500000 francs CFA de la pirogue "I. La plupart sont aujourd'hui dans une situation plus précaire qu'avant leur départ : "Au retour, je suis resté quatre mois sans rien faire, explique Souleymane ; i'étais fatigué, j'avais le cceur gros. Finalement, je suis resté à Dakar, je travaille comme journalier peintre et de temps en temps j'envoie de l'argent à la famille restée ò $T$. [un village de Casamance] ").

Bien souvent, ces réadmissions, loin d'engager un processus de développement, ont participé à accroître la pauvreté des migrants et de leur famille. Face aux réactions de l'opinion publique sénégalaise, elles ont été interrompues depuis 2008.

\section{- Des projets de "coopération" fragilisés par des desseins électoraux}

Par la suite, l'Espagne a tenté de promouvoir un système de quotas pour "l'attribution de contrats au profit de travailleurs sénégalais ". En 2007, une agence de recrutement a été créée avec la participation 
du Bureau intemational du travail (BT) et 5000 emplois ont été annoncés à terme. La sélection des candidats revient aux autorités sénégalaises et suscite beaucoup de polémiques au sein de la population. Pour le moment, seules quelques dizaines d'hommes, suivis par un petit contingent de femmes, ont bénéficié de ces mesures. Pourtant, la demande et l'offre persistent. En 2007, l'Espagne a proposé 105 emplois de pêcheurs; la Direction de l'emploi du Sénégal a décliné l'offre en invoquant le manque de main-d'œuvre disponible. Quelques mois plus tard, lors d'une manifestation à l'initiative des marchands ambulants des rues de Dakar, on pouvait lire sur les pancartes brandies par des pêcheurs : "L'Espagne a besoin de marins, le Sénégal a des marins I). La mise en œuvre de la politique dite des quotas semble avoir pâti d'un clientélisme politique, notamment en période électorale.

Les programmes de développement, le plan Retour vers l'agriculture (REVA) ou les "pôles de compétitivité "), soutenus par la coopération espagnole, se heurtent aux mêmes difficultés. Ces projets agricoles " ne répondent ni à une vraie perspective de développement ni à une demande formulée par les migrants de retour ", reconnaît un fonctionnaire européen en poste au Sénégal. Le "pôle de compétitivité ", implanté dans la région de Mbour, au sud de Dakar, est en cela révélateur : il s'agit d'un projet de culture de melons, entamé dans une zone de pâturage et destiné à des populations urbaines dont l'expérience professionnelle est éloignée de la culture maraîchère. Le choix de sa localisation a largement été guidé par des contingences politiques à la veille des élections locales.

Tous ces exemples soulignent les difficultés de mise en œurre des accords bilatéraux de coopération, promus par l'Espagne ou par d'autres pays européens, " en matière de régulation et de gestion des flux migratoires 1). Au-delà des discours tenus par les représentants des États signataires, souvent plus préoccupés par des enjeux électoraux que par les véritables enjeux du développement, les termes de ces accords sont emblématiques de la volonté de l'Europe de maintenir les candidats à l'émigration dans leur pays d'origine, d'inciter les pays de transit à réadmettre les migrants en situation irrégulière dans l'Union européenne et à prendre en charge leur renvoi vers les pays d'origine ou d'aider les pays de transit à se transformer en poste-frontière avancé.

À l'évidence, cette "coopération" est plus proche de l'esprit du Pacte européen sur l'immigration et l'asile, adopté par les 27 États de l'Union européenne le 16 octobre 2008, que des résolutions du Plan d'action de la Conférence euro-africaine Migration et développement, organisée à

Vol. $21, n^{\circ} 125$ septembre-octobre 2009 
Rabat en juillet 2006. En ce sens, lors de l'ouverture de la Réunion préparatoire à la Deuxième conférence ministérielle euro-africaine sur la migration et le développement pour la mise en place du Plan d'action de Rabat (9-1 1 juillet 2008), le ministre des Affaires étrangères du Sénégal a fait part de sa "I surprise devant l'initiative européenne d'un Pacte alors que le Processus de Rabat engageait [les parties] dans une autre voie 11.

\section{En guise de conclusion}

Le représentant de la diplomatie sénégalaise poursuivait en ces termes: "Si tout cela est confirmé, il sera urgent pour la partie africaine de se concerter et de se démarquer nettement des partenaires européens et de promouvoir fermement notre vision d'une migration concertée et non choisie et d'une approche globale et non ciblée 11.

L'un des préalables à cette initiative serait peut-être de déconstruire la notion de "subsaharien" et de prendre en considération la diversité de situations que ce terme revêt, y compris au sein des groupes nationaux.

Les politiques promues par l'Europe sont standardisées et appliquées à l'identique dans des contextes économiques et politiques aussi divers que complexes. Comment aborder de la même manière l'émigration du Nigeria, pays le plus peuplé d'Afrique, celle de la Guinée ou de la Mauritanie, théâtres de récents coups d'État, et celle du Ghana, dont l'économie bénéficie de la consolidation du processus démocratique? En outre, la diversité de ces environnements renvoie à des réalités démographiques et sociologiques profondément différentes: des femmes nigérianes, exploitées par l'industrie du sexe sur les trottoirs des métropoles européennes, aux modou-modou ${ }^{45}$ sénégalais, étroitement liés à la confrérie musulmane mouride, en passant par les petits-fils des premiers ouvriers maliens de l'industrie automobile française.

Dans ce contexte, si l'émigration ouest-africaine se fonde encore sur des causes économiques, ces dernières se combinent désormais à des causes politiques, liées aux conflits et aux reauls démocratiques qui affectent un nombre croissant d'États ouest-africains, le tout engendrant des causes sociales. Aujourd'hui, l'impossibilité d'émigrer suscite elle-même des mouvements de contestation dans les pays d'origine, les peuples manifestant leur désespérance de ne pouvoir émigrer. En novembre 2007, les titres des journaux sénégalais - "Dakar à feu et à sang ", "Émeutes

45. Commerçants ambulants. 
d'une rare intensité "), "Une folle journée d’émeutes jamais vues " - mettaient en exergue l'ambiance d'insurrection urbaine dans les rues de la capitale, où plusieurs centaines de vendeurs ambulants, chassés des trottoirs par la police, ont violemment protesté et ont été rejoints par des pêcheurs exprimant leur colère contre la rigueur des politiques migratoires européennes.

Ainsi, l'expression " Barsa ou Barça ", " Barcelone ou la mort ", scandée dans l'enceinte même du tribunal de Dakar par des migrants lors de leur comparution, est devenue le symbole de l'émigration ouest-africaine vers les îles Canaries. Elle fait écho au constat d'Adrian Adams qui écrivait dès 1977 que "l lorsqu'il faut émigrer pour vivre, on en vient inexorablement à vivre pour émigrer $11^{46}$.

46. ADAMS, Adrian, Le long voyage des gens du Flewve, Paris : Éd. Maspero, 1977, 222 p. (voir p. 129).

Vod. $21, n^{\circ} 125$

septembre - octobre 2009 